Invariant subspaces of the periodic Navier-Stokes and magnetohydrodynamics equations: Symmetries and inverse cascades

Manuel Núñez

Citation: Journal of Mathematical Physics 41, 6193 (2000); doi: 10.1063/1.1287920

View online: https://doi.org/10.1063/1.1287920

View Table of Contents: http://aip.scitation.org/toc/jmp/41/9

Published by the American Institute of Physics 


\title{
Invariant subspaces of the periodic Navier-Stokes and magnetohydrodynamics equations: Symmetries and inverse cascades
}

\author{
Manuel Núñez \\ Departamento de Análisis Matemático, Universidad de Valladolid, \\ 47005 Valladolid, Spain
}

(Received 14 February 2000; accepted for publication 24 May 2000)

It is shown that when the initial condition and the forcing term of the periodic Navier-Stokes or magnetohydrodynamics equations have Fourier coefficients which vanish outside a certain semigroup of frequencies, the same happens to the solutions for all time. Subgroups of frequencies correspond to solutions possessing certain symmetries. By taking as a semigroup the frequencies whose Fourier components are non-negative integers, we get a class of solutions for which the higher modes do not influence the evolution of the lower ones; therefore, the phenomenon of inverse cascading cannot occur for them. (C) 2000 American Institute of Physics. [S0022-2488(00)03609-4]

\section{INTRODUCTION}

So far there exists a wide gulf between what is accepted in fluid mechanics and what can be rigorously deduced from the Navier-Stokes equations. This is specially true in the field of turbulence, where the standard view of the transfer of energy in fully developed turbulent flows remains analytically unproved. Let use remember that energy is typically introduced into the turbulent fluid by a large-scale forcing, is transported to smaller scales through an intermediate (called inertial) range, and finally it is dissipated into heat at the dissipative range. For periodic problems, these ranges are identified with certain subsets of Fourier modes: the scales decrease as the spatial frequency grows. For dimension two, however, the above picture of a direct cascade is not entirely correct: Kraichnan and others ${ }^{1-5}$ showed that energy injected at a certain intermediate scale may be transferred to the largest scales and fairly obvious large structures appear in the flow. Those inverse cascades are believed to occur also for a number of magnetohydrodynamic magnitudes: the mean-square magnetic potential in two-dimensional and the magnetic helicity in threedimensional magnetohydrodynamics (MHD) (see Ref. 6 for the relevant definitions).

The standard modern presentation ${ }^{7-10}$ of these evolution equations involves a number of subspaces of the space of square-integrable functions $L^{2}(\Omega)$, plus Galerkin approximations and compactness theorems. These techniques work as well for closed subspaces of the standard ones, provided these subspaces are invariant for the relevant operators. For the periodic case, a number of possibilities are apparent. By choosing a certain class of invariant subspaces, we will show that when the initial condition and the forcing term satisfy a certain type of symmetry, the same happens to the solutions for as long as they are defined. A second example will yield whole families of solutions for which there is no inverse cascade at all: the large scales ignore the small ones in their evolution, irrespective of the value of the Reynolds number. These results, however, do not represent a threat for our picture of inverse cascading, because our velocity and magnetic fields are complex functions and therefore do not have any clear-cut physical meaning. The nonlinear character of the Navier-Stokes and MHD equations precludes the classical trick of taking the real part of a complex solution to find a real one. Hopefully this construction may be helpful to understand which analytic techniques may work in order to prove rigorously the existence of inverse cascades. Another caveat is that our results depend on very specific arithmetical 
properties of the complex exponentials and therefore cannot be extended in any obvious way to other boundary value problems.

\section{THE MATHEMATICAL FORMALISM}

Consider a viscous incompressible fluid filling (for notational simplicity) the box $\Omega$ $=[0,2 \pi]^{N}$, possessing a velocity $\mathbf{u}$ and pressure $p$, under the action of a time-independent forcing f. We will assume that all the magnitudes are spatially periodic, and that the mean of $\mathbf{u}$ and (necessarily) $\mathbf{f}$ are zero,

$$
\langle\mathbf{u}\rangle=\int_{\Omega} \mathbf{u}(t, \mathbf{x}) d V=\mathbf{0} .
$$

After normalization of constants, $\mathbf{u}$ will satisfy the incompressible Navier-Stokes equation

$$
\begin{gathered}
\frac{\partial \mathbf{u}}{\partial t}=\nu \Delta \mathbf{u}-\mathbf{u} \cdot \nabla \mathbf{u}-\nabla p+\mathbf{f}, \\
\nabla \cdot \mathbf{u}=0, \\
\mathbf{u}(0)=\mathbf{u}_{0},
\end{gathered}
$$

where $\nu$ stands for the fluid viscosity. If the fluid is a conducting one with resistivity $\eta$, and a magnetic field $\mathbf{B}$ is present, it acts upon the velocity through the Lorentz force while satisfying the induction equation. The resulting magnetohydrodynamic (MHD) equations, after normalization of constants, are

$$
\begin{gathered}
\frac{\partial \mathbf{u}}{\partial t}=\nu \Delta \mathbf{u}-\mathbf{u} \cdot \nabla \mathbf{u}+\mathbf{B} \cdot \nabla \mathbf{B}-\nabla\left(p+\frac{B^{2}}{2}\right)+\mathbf{f}_{1}, \\
\frac{\partial B}{\partial t}=\eta \Delta \mathbf{B}-\mathbf{u} \cdot \nabla \mathbf{B}+\mathbf{B} \cdot \nabla \mathbf{u}+f_{2}, \\
\nabla \cdot \mathbf{u}=0, \\
\nabla \cdot \mathbf{B}=0, \\
\langle\mathbf{u}\rangle=\langle\mathbf{B}\rangle=\mathbf{0}, \\
\mathbf{u}(0)=\mathbf{u}_{0}, \\
\mathbf{B}(0)=\mathbf{B}_{0} .
\end{gathered}
$$

Both systems may be cast in the same form: after projection in the space of fields with zero divergence (killing in this way all the gradients), one gets

$$
\frac{\partial \mathbf{w}}{\partial t}=D \mathbf{w}+C(\mathbf{w}, \mathbf{w})+\mathbf{f},
$$

where $\mathbf{w}=\mathbf{u}$ (Navier-Stokes) or $(\mathbf{u} ; \mathbf{B})(\mathrm{MHD}) ; D$ is an elliptic operator (either $v \Delta$ or $(v \Delta ; \eta \Delta))$, and $C$ is a bilinear form with the antisymmetric property $\left(C\left(\mathbf{w}_{1}, \mathbf{w}_{2}\right), \mathbf{w}_{2}\right)=0$. For the NavierStokes equations, $C(\mathbf{u}, \mathbf{v})=-P(\mathbf{u} \cdot \nabla \mathbf{v})$, where as mentioned before $P$ is the orthogonal projection upon the space of solenoidal fields, with mean zero. In the MHD case,

$$
C\left(\left(\mathbf{u}_{1}, \mathbf{B}_{1}\right) ;\left(\mathbf{u}_{2} ; \mathbf{B}_{2}\right)\right)=\left(P\left(-\mathbf{u}_{1} \cdot \nabla \mathbf{u}_{2}+\mathbf{B}_{1} \cdot \nabla \mathbf{B}_{2}\right) ; P\left(-\mathbf{u}_{1} \cdot \nabla \mathbf{B}_{2}+\mathbf{B}_{1} \cdot \nabla \mathbf{u}_{2}\right)\right) .
$$


From now on, we will restrict ourselves to the Navier-Stokes equations to simplify the notation. The proofs for the MHD case are the same with obvious modifications.

The main working spaces are

$$
\begin{gathered}
H=\left\{\mathbf{u} \in L^{2}(\Omega)^{N}: \nabla \cdot \mathbf{u}=0,\langle\mathbf{u}\rangle=\mathbf{0}, \mathbf{u} \cdot \mathbf{n} \text { anti-periodic }\right\}, \\
V=H^{1}(\Omega)^{N} \cap H .
\end{gathered}
$$

The divergence of $\mathbf{u}$ is to be understood in the sense of distributions. It may be shown that the boundary values $\mathbf{u} \cdot \mathbf{n}$ have in this case a precise mathematical sense. ${ }^{7} H^{1}(\Omega)$ is the usual Sobolev space of functions whose gradient is square-integrable. The projection $P: L^{2}(\Omega)^{N} \rightarrow H$ is easily expressed in terms of the Fourier series of a periodic function,

$$
P\left(\sum_{\mathbf{k} \in Z^{N}} \mathbf{a}_{\mathbf{k}} e^{i \mathbf{k} \cdot \mathbf{x}}\right)=\sum_{\mathbf{k} \in \mathbb{Z}^{N}} \mathbf{b}_{\mathbf{k}} e^{i \mathbf{k} \cdot \mathbf{x}},
$$

where $\mathbf{b}_{\mathbf{0}}=\mathbf{0}$, and $\mathbf{b}_{\mathbf{k}}$ is the projection within $\mathrm{C}^{N}$ of $\mathbf{a}_{\mathbf{k}}$ on the hyperplane $\mathbf{k} \cdot \mathbf{x}=0$. It is known that $C$ takes $V \times V$ into the dual space $V^{\prime}$.

\section{ALTERNATIVE SETTINGS AND CONSEQUENCES}

Let $H_{0}$ be a closed subspace of $H$ such that the Laplacian $\Delta$ takes $H^{2}(\Omega)^{N} \cap H_{0}$ into $H_{0}$, and if $\mathbf{u}, \mathbf{v} \in H_{0}$ are such that $P(\mathbf{u} \cdot \nabla \mathbf{v}) \in H$, then $P(\mathbf{u} \cdot \nabla \mathbf{v}) \in H_{0}$. Let $V_{0}=V \cap H_{0}$, and assume $\mathbf{u}_{0}, \mathbf{f}$ $\in H_{0}$. Then all the standard proofs of existence work with $H_{0}, V_{0}$ instead of $H, V$ and we get the same results: the solution $\mathbf{u} \in \mathcal{C}\left([0, T], H_{0}\right) \cap L^{2}\left([0, T], V_{0}\right)$ up to some $T(T=\infty$ for $N=2)$ and, if $\mathbf{u}_{0} \in V_{0}, \mathbf{u} \in \mathcal{C}\left([0, T], V_{0}\right) \cap L^{2}\left([0, T], H_{0} \cap H^{2}(\Omega)^{N}\right)$. Thus the solution remains within $H_{0}$ for all time. The only difficulty is to find a subspace $H_{0}$ satisfying all the requirements. Let us look at the Fourier representation of $\mathbf{u}$. $\Delta \mathbf{u}$ corresponds to $\Sigma-|\mathbf{k}|^{2} \hat{\mathbf{u}}_{\mathbf{k}} e^{i \mathbf{k} \cdot \mathbf{x}}$. On the other hand, the $k$-th Fourier coefficient of $u_{j} v_{l}$ is

$$
\left\langle u_{j} v_{l}\right\rangle(\mathbf{k})=\sum_{\mathbf{n} \in \mathbb{Z}^{N}} \hat{u}_{j}(\mathbf{n}) \hat{v}_{l}(\mathbf{k}-\mathbf{n}) .
$$

Take tentatively as $H_{0}$ the space of functions of $H$ whose Fourier coefficients vanish outside a subset $A \subset Z^{N}$. Then $H_{0}$ is invariant for $\Delta$. For the product of two functions of $H_{0}$ to lie within $H_{0}$, we need that whenever $\mathbf{k} \notin A,\left(u_{j} v_{l}\right)^{\wedge}(\mathbf{k})=0$. Since we may choose at will $u_{j}$ and $v_{l}$, this happens if and only if all the summands within (8) vanish, i.e., if $\mathbf{k} \notin A, \mathbf{n} \in A$, then $\mathbf{k}-\mathbf{n} \notin A$. This means that $A$ must be a semigroup of $Z^{N}$ : i.e., if $\mathbf{m}, \mathbf{n} \in A$, then $\mathbf{m}+\mathbf{n} \in A$. The projection $P$ keeps such functions within $H_{0}$, since it does not extend the support of the Fourier transform. Hence any subspace $H(A)$ formed by the functions whose Fourier transform has support contained within a semigroup $A \subset Z^{N}$ is an invariant one for the Navier-Stokes equation.

If $A$ is a subgroup, we may say a good deal about the possible solutions. Subgroups of $Z^{N}$ are free and generated by at most $N$ elements. This means that there are a number of basic frequencies, $\mathbf{k}_{1}, \ldots \mathbf{k}_{p}, p \leqslant N$, such that $H(A)$ is formed by the functions whose Fourier modes correspond to frequencies which are a linear combination with integer coefficients of $\mathbf{k}_{1}, \ldots \mathbf{k}_{p}$. Since any complex exponential $e^{i n \mathbf{k} \cdot \mathbf{x}}$ is a function of $\mathbf{k} \cdot \mathbf{x}$, such solutions depend only on the variables $\mathbf{k}_{1}$ $\cdot \mathbf{x}, \ldots \mathbf{k}_{p} \cdot \mathbf{x}$. To see the meaning of this, consider $N=2, \mathbf{k}_{1}=(1,0)$. All such velocities are functions of the first spatial variable $x$. Take now $\mathbf{k}_{1}=(2,0), \mathbf{k}_{2}=(0,2)$. Then the velocities depend on $2 x, 2 y$, i.e., $\mathbf{u}(x, y)=\mathbf{u}(\pi+x, y)=\mathbf{u}(x, \pi+y)$. We see that those are functions satisfying a certain number of symmetries. It is not unexpected for the Navier-Stokes and MHD equations to keep for all time the symmetries of the initial condition and the forcing, although it is not entirely trivial given their nonlinear character. 
The analysis for general semigroups is made difficult by its possible complicated structure. We will concentrate on the simplest and most important: $\mathbb{N}^{N}$, i.e., the set of $\mathbf{k} \in \mathbb{Z}^{N}$ with $k_{j} \geqslant 0$ for all $j$. Of course, if $A$ is a semigroup, so is $-A$, which means that we could have taken as well negative frequencies.

Let $Q_{M}$ denote the projection from $L^{2}(\Omega)^{N}$ into the finite-dimensional space of trigonometric polynomials with frequencies bounded by $M$,

$$
\begin{gathered}
Q_{M}(f)=\sum_{|\mathbf{k}| \leqslant M} \hat{f}(\mathbf{k}) e^{i \mathbf{k} \cdot \mathbf{x}}, \\
|\mathbf{k}|=\left|k_{1}\right|+\cdots+\left|k_{N}\right| .
\end{gathered}
$$

Let $\mathbf{u}$ be a solution of the Navier-Stokes equations such that $\mathbf{u}_{0}, \mathbf{f} \in H\left(\mathbb{N}^{N}\right)$. Then $Q_{M} \mathbf{u}$ satisfies

$$
\begin{gathered}
\frac{\partial Q_{M} \mathbf{u}}{\partial t}=\nu \Delta Q_{M} \mathbf{u}-P Q_{M}\left(Q_{M} \mathbf{u} \cdot \nabla Q_{M} \mathbf{u}\right)+Q_{M} \mathbf{f}, \\
\nabla \cdot Q_{M} \mathbf{u}=0, \\
Q_{M} \mathbf{u}(0)=Q_{M} \mathbf{u}_{0} .
\end{gathered}
$$

The proof is simple: let us apply the projection $Q_{M}$ to the Navier-Stokes equation. $Q_{M}$ commutes with $\partial / \partial t, \Delta$, and $P$. For any product $u v$ of functions within $H\left(\mathbb{N}^{N}\right)$, and $|\mathbf{k}| \leqslant M$, the kth Fourier coefficient is

$$
(u v)^{\wedge}(\mathbf{k})=\sum_{\mathbf{l}} \hat{u}(\mathbf{l}) \hat{v}(\mathbf{k}-\mathbf{l})
$$

For any summand to be nonzero, we need $l_{j} \geqslant 0, k_{j}-l_{j} \geqslant 0$, which means $k_{j} \geqslant 0$, plus $\Sigma_{j} k_{j} \leqslant M$; thus $\sum_{j} l_{j} \leqslant M, \sum_{j} k_{j}-l_{j} \leqslant M$. Hence only the coefficients $\hat{u}(\mathbf{l}), \hat{v}(\mathbf{l})$ with $|\mathbf{l}| \leqslant M$ occur. In other words,

$$
Q_{M}(\mathbf{u} \cdot \nabla \mathbf{u})=Q_{M}\left(Q_{M} \mathbf{u} \cdot \nabla Q_{M} \mathbf{u}\right)
$$

from which the result follows.

Equation (10) is in fact an ordinary differential one, since $Q_{M} \mathbf{u}$ is finite dimensional: all the spatial derivatives are continuous linear functionals. It is known that it has a unique solution for all time (even when $N=3$ ) because the nonlinear term is a polynomial in $Q_{m} \mathbf{u}$ : it is a finitedimensional reaction-diffusion equation. Standard energy inequalities show that $Q_{m} \mathbf{u}$ tends in $L^{2}((0, \infty), H)$ to a weak solution $\mathbf{u}$, which of course coincides for $N=2$ with the smooth one.

The important thing, however, is that $Q_{M} \mathbf{u}$ satisfies by itself an evolution equation with no implication whatsoever of the remaining modes of $\mathbf{u}$. Thus, although smaller frequencies (larger scales) do have an effect on the behavior of a certain mode, larger frequencies (smaller scales) do not. This clearly precludes inverse cascades.

As for the kind of forcing within $H\left(\mathbb{N}^{N}\right)$, they can be indeed very complicated functions, but they cannot be real ones: for real functions one has $\hat{\mathbf{f}}(-\mathbf{k})=\overline{\hat{\mathbf{f}}(\mathbf{k})}$. Hence $\mathbf{u}$ itself must be complex, which is hard to interpret as a physically realistic velocity field. And, as told before, the real part of $\mathbf{u}$ is not a solution of a Navier-Stokes equation with time-independent forcing: certainly one could add a term $-\mathbf{v} \cdot \nabla \mathbf{v}$ to $\mathbf{f}$, with $\mathbf{v}$ the imaginary part of $\mathbf{u}$, but of course any function is a solution of the Navier-Stokes equation with a suitable forcing term, so this is not meaningful. Moreover, this trick would yield a time-dependent forcing term.

It is worth noting that when $\mathbf{f}$ satisfies a Gevrey condition $\left(\Sigma|\hat{\mathbf{f}}(\mathbf{k})|^{2} e^{\sigma|k|}<\infty\right.$ for some $\sigma$ $>0$ ), the solution $\mathbf{u}$ is itself Gevrey for as long as it remains bounded in the $H^{1}$-norm, which mean always for $N=2$ (see Ref. 11; the proofs there work without substantial modification for the 
MHD case). Taking as new variables $z_{j}=e^{i x_{j}}$, this means that $\mathbf{f}$ and $\mathbf{u}(t)$ may be analytically extended to a neighborhood of the torus $T^{N} \subset \mathrm{C}^{N}$. If moreover $\mathbf{f}$ and $\mathbf{u}_{0}$ belong to $H\left(\mathbb{N}^{N}\right)$, the Fourier expansion becomes a Taylor one, and what we have proved is that $\mathbf{u}(t)$ remains analytic in a neighborhood of the polidisk $\bar{B}(\mathbf{0}, 1) \subset \mathrm{C}^{N}$. The wide variety of analytic functions shows that the forcing functions $\mathbf{f}$ may be made to satisfy many properties.

Concerning other magnitudes for which the phenomenon of inverse cascading is claimed, we find much the same result. The mean-square magnetic potential in two-dimensional MHD is found in the following way: let $\mathbf{B}=\Sigma \hat{\mathbf{B}}(\mathbf{k}) e^{i \mathbf{k} \cdot \mathbf{x}}$ be the Fourier series of the plane magnetic field $\mathbf{B}$ $=\left(B_{1}, B_{2}, 0\right)$, satisfying as always $\mathbf{k} \cdot \hat{\mathbf{B}}(\mathbf{k})=0$. Take $\hat{A}(\mathbf{k})$ such that $i \mathbf{k} \times(0,0, \hat{A}(\mathbf{k}))=\hat{\mathbf{B}}(\mathbf{k})$. Then $A(\mathbf{x})=\Sigma \hat{A}(\mathbf{k}) e^{i \mathbf{k} \cdot \mathbf{x}}$ is the scalar potential vector. Its mean-square potential is $\Sigma|\hat{A}(\mathbf{k})|^{2}$. Since the Fourier components $\hat{\mathbf{B}}(\mathbf{k}),|\mathbf{k}| \leqslant M$, depend for all time only on the remaining $\hat{\mathbf{B}}(\mathbf{l}),|\mathbf{l}| \leqslant M$, the same happens for $\hat{A}(\mathbf{k})$. Thus the mean potential of $Q_{M} A$ depends only on the previous history of the modes up to size $M$, and there is no inverse cascade. As for the magnetic helicity $\mathcal{H}$ in three dimensions, it has no interest in $H\left(\mathbb{N}^{N}\right)$ because $\mathcal{H}=\Sigma \hat{\mathbf{A}}(\mathbf{k}) \cdot \hat{\mathbf{B}}(-\mathbf{k})=0$. Since the magnetic helicity measures in a sense the knottedness of the magnetic field, these fields are in a sense topologically simple; but this has not much to do with turbulence, which may be present at dimension two, where there is no possibility of a field to become entangled.

\section{CONCLUSIONS}

By analyzing the invariant subspaces of the operators occurring in the periodic Navier-Stokes and magnetohydrodynamic equations, we have found that when the Fourier frequencies of the forcing and initial conditions lie within a certain semigroup of $\mathbb{Z}^{N}$, the same happens to the solution for as long as it is defined in the classical sense. When we take as a semigroup a subgroup of $Z^{N}$, we find that these solutions correspond to fields satisfying a certain number of symmetries. By taking as a semigroup the set of positive frequencies, we find that the truncated Fourier series of the solution satisfies an evolution equation by itself and therefore it ignores the remaining Fourier modes. As a consequence there cannot be inverse cascading for this class of solutions. These turn out to be always complex functions and not realistic physical fields, but these results may be helpful to understand the analytic setting where existence of inverse cascades may eventually be rigorously proved.

${ }^{1}$ R. H. Kraichnan, “'Inertial ranges in two-dimensional turbulence," Phys. Fluids 10, 1417-1423 (1967).

${ }^{2}$ R. H. Kraichnan, "Eddy viscosity in two and three dimensions," J. Atmos. Sci. 33, 1521-1536 (1976).

${ }^{3}$ E. D. Siggia and H. Aref, "Point-vortex simulation of the inverse cascade in two-dimensional turbulence," Phys. Fluids 24, 171-173 (1981).

${ }^{4}$ U. Frisch and P.-L. Sulem, "Numerical simulation of the inverse cascade in two-dimensional turbulence," Phys. Fluids 27, 1921-1923 (1984).

${ }^{5}$ V. Borue, "Inverse energy cascade in stationary two-dimensional turbulence," Phys. Rev. Lett. 72, 1475-1478 (1994).

${ }^{6}$ D. Biskamp, Nonlinear Magnetohydrodynamics (Cambridge University Press, Cambridge, 1993).

${ }^{7}$ R. Temam, Navier-Stokes Equations, Theory and Numerical Analysis (North-Holland, Amsterdam, 1984).

${ }^{8}$ R. Temam, Navier-Stokes Equations and Nonlinear Functional Analysis, CBMS-NSF Regional Conference Series in Applied Mathematics, SIAM, Philadelphia, 1983.

${ }^{9}$ M. Sermange and R. Temam, "Some mathematical questions related to the MHD equations," Commun. Pure Appl. Math. 36, 635-664 (1983).

${ }^{10}$ R. Temam, Infinite Dimensional Dynamical Systems in Mechanics and Physics (Springer, New York, 1988).

${ }^{11}$ C. Foias and R. Temam, "Gevrey class regularity for the solutions of the Navier-Stokes equations,'” J. Funct. Anal. 87, 359-369 (1989). 